\title{
Knowledge and Attitudes of Nursing Students Towards Smokeless Tobacco and Areca Nut Control in Central Province of Sri Lanka
}

\author{
PVKS Hettiarachchi ${ }^{1,2}$, Primali Jayasooriya ${ }^{2,3}$, Hemantha Amarasinghe ${ }^{2,4}$, BSMS \\ Siriwardena ${ }^{2,3}$, Deepashika Wijerathne ${ }^{2}$, Samantha Kumara Kithalawaarachchi ${ }^{2}$, \\ WM Tilakaratne ${ }^{2,3,5}$, Ruwan D Jayasinghe ${ }^{1,2}$
}

${ }^{1}$ Department of Oral Medicine and Periodontology, Faculty of Dental sciences, University of Peradeniya, Sri Lanka, ${ }^{2}$ Centre for Research in Oral Cancer, Faculty of Dental Sciences, University of Peradeniya, Sri Lanka. ${ }^{3}$ Department of Oral Pathology, Faculty of Dental Sciences, University of Peradeniya, Sri Lanka. ${ }^{4}$ Training Unit, Institute of Oral Health, Maharagama, Sri Lanka. ${ }^{5}$ Department of Oral and Maxillofacial Clinical Sciences, Faculty of Dentistry, University of Malaya, Malaysia.

\begin{abstract}
Background: Use of smokeless tobacco (SLT) and areca nut (AN) is widespread in Sri Lanka. Several workshops to train healthcare workers on SLT/ AN cessation programmes (SLT/AN-CP) were carried out. Objective of the study was to evaluate the enhancement of knowledge and attitudes related to SLT/AN-CP among nursing students following a workshop. Methods: A cross sectional study was conducted with the use of two questionnaires. A total of 212 nursing students were assessed and the study group included 184 females (86.8\%). Results: Results were analyzed according to the 3 main areas of the assessment, i.e knowledge related to Oral cancer, knowledge related to SLT, its effects and cessation practices and attitudes regarding SLT cessation counselling. Out of the eight questions to assess the knowledge related to oral cancer, five questions demonstrated a statistically significant difference $(\mathrm{P}<0.05)$ following the workshop when compared to the pre workshop knowledge. Even though, majority had a good knowledge on harmful effects of SLT and AN, it was interesting to note that $14.15 \%$ of the participants were unaware about the fact that the oral cancer is the commonest cancer among Sri Lankan males. $96.7 \%$ agreed that proper counseling would lead patient to quit the habit. Conclusions: This preliminary study showed that the knowledge and attitude towards SLT/AN-CP among nursing students were satisfactory and effective workshops can be used to improve their knowledge and attitudes towards SLT/AN-CP especially in low economical settings.
\end{abstract}

Keywords: Smokeless tobacco- arecanut- tobacco cessation- nursing students

Asian Pac J Cancer Care, 5 (3), 133-138

Submission Date: 05/03/2020Ａcceptance Date: 07/02/2020

\section{Introduction}

Tobacco is one of the greatest threats to global health today. Use of both smoking and smokeless tobacco (SLT) is associated with increased risk of chronic and terminal diseases. These encompass periodontal diseases, oral and pharyngeal cancers, myocardial infarction, stroke, erectile dysfunction and problems in pregnancy, leading to stillbirths and low birth weight babies [1]. SLT is an addiction for millions of people worldwide, and research indicates that the use by young individuals in many countries is increasing. The magnitude of this problem in Sri Lanka was showed in a recent survey, where $15.8 \%$ of Sri Lankans which includes $8.6 \%$ of the youth have been estimated to be smokeless tobacco (SLT) users [2]. SLT users outnumber the estimated number of smokers in many countries of the region including Sri Lanka; the prevalence of smoking is decreasing, while the use of SLT is on the rise [3]. A variety of types of SLT are consumed throughout the world and these include betel

\section{Corresponding Author:}

Dr. Kalani Hettiarchchi

Department of Oral Medicine and Periodontology, Faculty of Dental Sciences, University of Peradeniya, Sri Lanka.

Centre for Research in Oral Cancer, Faculty of Dental Sciences, University of Peradeniya, Sri Lanka.

Email: kalaniz2004@yahoo.com 
quid and other commercially available tobacco products such as pan masala, mawa, red tooth powder, khaini, tobacco powder, zarda and many more [2].

The harmful effects of SLT use has been well documented and includes oral squamous cell carcinoma (OSCC) at the dubious first place producing the highest incidence and highest mortality out of all cancers among Sri Lankan males [4]. However, it is unfortunate that successful SLT cessation remains a weak point in tobacco control in the South-East Asia Region. Though there is clear scientific evidence that tobacco cessation interventions are highly cost-effective public health measures, countries of the region have failed to optimally prioritize this issue so far and therefore, programmes are yet to be developed to eradicate this preventable menace [5-6].

Majority of patients with OSCC is preceded by oral potentially malignant disorders (OPMD) [5-8]. According to the management protocols of OPMD established in Sri Lanka [9] low risk OPMDs are managed at primary care level by general dental practitioners with education on habit intervention with 6 month review appointments. Thus, Dental professionals are well suited and can play a major role in tobacco cessation activity [10-13]. However, the National Institute for Health and Care Excellence (NICE) (2012) guideline for Smokeless tobacco recommends the involvement of primary and secondary dental care teams (example, dentists, dental nurses and dental hygienists) for providing brief advice and referral in South Asian communities [14]. Therefore, in par with these professionals other than the dentists, nursing officers can play an important role in tobacco cessation (TC) activities. Majority of tobacco cessation protocols have been developed with smoking cessation in mind [15-16]. However, though the same protocols can be applied for SLT cessation as well, successful implementation may require at least some modifications. Firstly, with reference to SLT, in addition to tobacco, cessation of areca nut use should also be targeted. Furthermore, dependency and withdrawal symptoms of SLT use as well as areca nut use show differences when compared with smoking [17-18]. Thus, a training programme on SLT cessation was developed to improve the knowledge of healthcare workers with reference to above mentioned facts.

Therefore, the objective of the present study was to assess the knowledge and attitudes of nursing students in relation to SLT/AN cessation and to assess the enhancement of knowledge, and attitudes following a programme on SLT/AN cessation.

\section{Materials and Methods}

Description of the workshops conducted for SLT / AN cessation (SLT / AN cessation programmes (SLT/AN-CP)

Island-wide workshops to train healthcare workers including nursing students on tobacco cessation activities were planned in collaboration with Centre for Research in Oral cancer, Presidential task force for Drug Prevention and National Cancer Control Programme. This programme included 2 workshops conducted for nursing students from the Central Province. The workshop was conducted for a half a day, and the resource personals were subject specialists with experience in tobacco and AN cessation.

\section{Methodology}

Following the ethical approval from the Ethics Review Committee of the Faulty of Dental Sciences, a cross sectional study was conducted among the nursing students in the Central province who participated in the workshop. Two self administered questionnaires were developed by the investigators, to evaluate the nursing officers' knowledge and attitudes towards tobacco cessation practices and oral cancer. The first questionnaire which was administered prior to the workshop consisted of four components and the summary of the questioner is given in Table 1.

Self administered questinnaire was developed in Sinhala language, forward and backward translations was done in to English and Tamil languages. Content validation for this questionnaire was carried out with the help of three Consultants in Community Dentistry. The content validity ratio per item was calculated and each item in the questionnaire had a CVR ratio (CVR) of $>0.75$ [8]. Pre-testing of questionnaire was conducted among ten nursing students from the Faculty of Dental Sciences, University of Peradeniya. The questionnaire was self administered on voluntary basis prior to the commencement of the workshop.

The second questionnaire contained all the components mentioned in Table 1 except the demographic profile of the patients and this was administered following completion of the workshop.

\section{Statistical analysis}

The SPSS (version 22) software package was used for the data analysis. Data obtained from the questionnaire were entered in SPSS software were expressed as frequencies (percentages) using descriptive statistics. The improvement of the knowledge following the workshop was assessed by comparing the pre vs. post workshop responses using McNemar test. Attitudinal questions were weighted and amalgamated to produce total attitudinal value for each subjects. Total values were converted to $\mathrm{Z}$ scores and dichotomized to unsatisfactory and satisfactory attitude. Pre and post dichotomized values were tested for significance by McNemar test. P-values of less than 0.05 were considered statistically significant.

\section{Results}

Participants who answered all items of the questionnaire were considered as complete responders and only completed questionnaires were analyzed. Out of the 212 nursing students who responded, 93 were undergraduates from the Faculty of Allied Health Sciences (AHS) and 119 was from the Nurses Training School (NTS), Kandy. The study group had a female preponderance with 184 females $(86.8 \%)$ and 28 males $(13.2 \%)$. The undergraduates from the Faculty of AHS 
Table 1. Summary of the Pre Workshop Questionnaire

\begin{tabular}{lcc}
\hline Components & No of Questions & \multicolumn{1}{c}{ Type of the questions } \\
\hline $\begin{array}{l}\text { 1. Demographic profile of the participants } \\
\text { (No data was collected on personal identifiers of the }\end{array}$ & 07 & Majority dichomatous questions with Some open ended \\
students) & & \\
$\begin{array}{l}\text { 2. Awareness and knowledge related to oral cancer } \\
\begin{array}{l}\text { 3. Knowledge related to smokeless tobacco, its } \\
\text { effects and cessation practices }\end{array}\end{array}$ & 08 & 3-point Likert scale (true, false, and Don't know) \\
$\begin{array}{l}\text { 4. Attitudes regarding smokeless tobacco cessation } \\
\text { counseling (TCC) and ways to reduce SLT use. }\end{array}$ & 05 & 3-point Likert scale (true, false, and Don't know) \\
\hline
\end{tabular}

m Correct Resopnse in Post workshop $=$ Correct Resopnse in Pre workshop

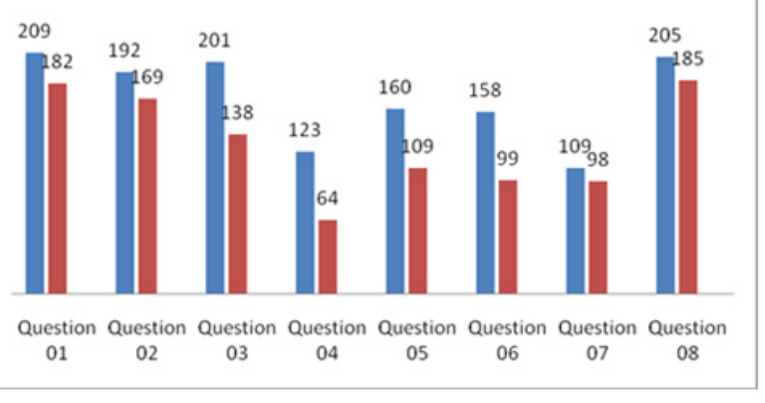

Figure 1. Graphical Representation of Participants who Indicated Correct Response to Each Question Allocated to Assess the "Awareness and Knowledge Related to Oral Cancer”

was within the age range of 20-25 years, however, the students age of NTS ranged from 20 to 50 years with $78.99 \%$ of the students of the NTS were in the age range of $20-25$ years. The majority $(66 \%)$ of the participants were in their 3rd year of the study programme. Only 23 $(10.8 \%)$ nursing students has received training on SLT/AN cessation before coming to the workshop and out of that $23,2.4 \%$ were in the opinion that the training they have received was adequate. Students who had a family history of oral cancer or had any immediate family member been diagnosed to have a disease (other than oral cancer) related to tobacco use was $1.9 \%$ and $4.7 \%$ respectively.

\section{Analysis of the Awareness and knowledge related to oral cancer \\ Out of the eight questions to assess the knowledge} related to oral cancer, five questions demonstrated a statistically significant difference $(\mathrm{P}<0.05)$ following the workshop when compared to the pre workshop knowledge. $14.15 \%$ of the participants were unaware of the fact that Oral cancer is the commonest cancer among Sri Lankan males. However, the knowledge was drastically improved with $98.58 \%$ acknowledged this fact immediately following the workshop and the results were statistically significant at a $\mathrm{P}$ value of $0.00(\mathrm{P}<0.05)$. Further, 34.9\% did not know that a longstanding white patch can be an oral cancer. It was noted that more than half of the participants, lack the knowledge on certain misconceptions such as oral cancer may occur due to spicy foods, oral cancer in the initial stages are painful and that all oral potentially malignant disorders invariably transform in to oral cancer were $68.9 \%, 53.3 \%$ and $53.8 \%$ respectively. They did not have a clear knowledge about the above mentioned facts and the number of correct answers received for these questions were statistically significant at a $\mathrm{P}$ value of $0.002,0.004$ and 0.00 .

Out of the 212 nursing students who participated, 46

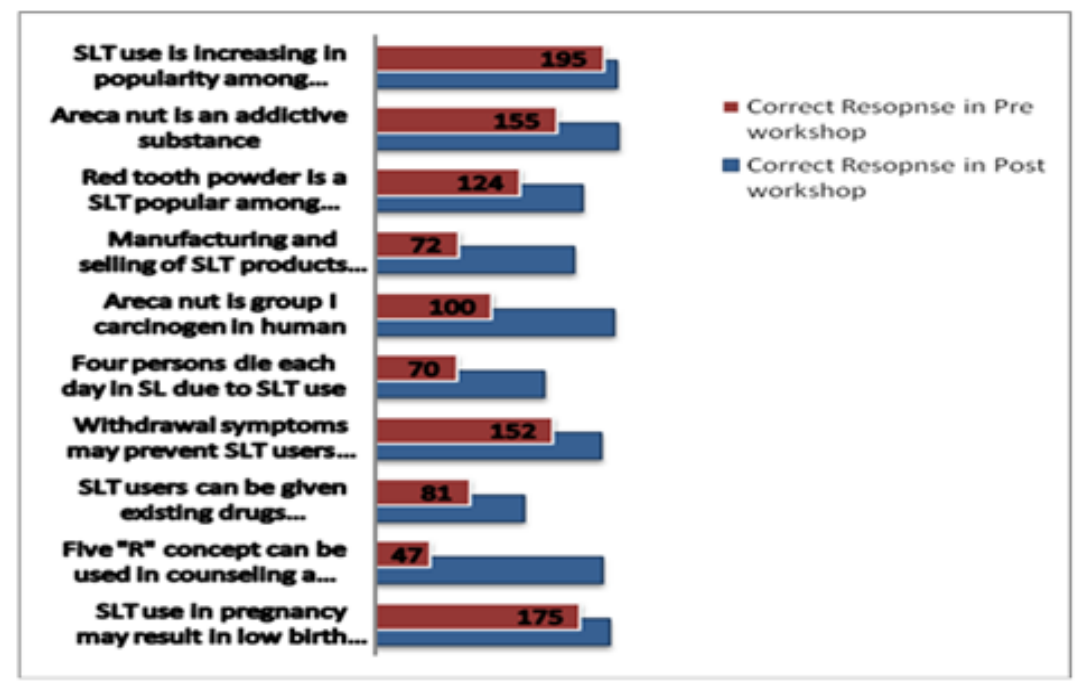

Figure 2. Graphical Representation of Participants who Indicated Correct Response to each Question Allocated to Assess the "Knowledge Related to Smokeless Tobacco, Its Effects and Cessation Practices" 
Table 2. Depicts the Pre and Post Dichotomized Attitude Scores

\begin{tabular}{lc}
\hline Pre test & Post test \\
& Satisfactory attitudes N (\%) \\
\hline Satisfactory attitudes N (\%) & $193(98)$ \\
Unsatisfactory attitude N (\%) & $4(2)$ \\
Total N (\%) & $197(100)$ \\
\hline
\end{tabular}

N- Number of questioners

$(21.7 \%)$ were on the opinion that majority of oral cancers cannot be prevented and the post workshop questionnaire showed a change in this opinion with 192 participants $(90.6 \%)$ stating that the statement as true with a P value of 0.092 which was not statistically significant. However, it was interesting to note that $87.3 \%$ knew that, oral cancer is curable if detected at the early stages.

Analysis of knowledge related to smokeless tobacco, its effects and cessation practices

As per the data obtained on the ten Likert scale questions, only four questions demonstrated a statistically significant difference $(\mathrm{P}<0.05) .195(92 \%)$ agreed upon the fact that SLT use is increasing in popularity among youth and adolescents, and 124 (58.5\%) were aware on the fact that "Red tooth powder" is a SLT product popular among children. This is an encouraging finding as these figures indicate public awareness. However, we found that only 155 participants $(73.1 \%)$ knew that "areca nut is an addictive substance" and the correct response between the two groups (pre and post workshop) were significantly different $(\mathrm{P}=0.021 ; \mathrm{P}<0.05)$. Further, it was also noted that only $72(33.9 \%)$ participants were aware of the fact that "manufacturing and selling of SLT products is banned in Sri Lanka" with a P value of 0.00 .

Further, majority $59.9 \%$ of the nursing students did not know the fact that "four persons die each day in Sri Lanka due to SLT use". Also, 73.1\% and 51.9\% respectively did not know that "SLT users can be given existing drugs prescribed to combat cigarette craving" and "Five " $\mathrm{R}$ " concept can be used in counseling a client who is unwilling to quit SLT use". Responses to 14 individual Likert scale items of the questionnaire have been depicted in Figure 2.

Analysis of the attitudes regarding smokeless tobacco cessation counseling (TCC) and ways to reduce SLT use

Out of the 212 participants only 197 has completely answered the section pertaining to attitudes, therefore, only those were considered for the assessment. The majority was in agreement that TCC is not effective due to lack of formal training $(79.7 \%)$ and that TCC should be part of the regular treatment modalities $(92.5 \%)$ and $96.7 \%$ believed that proper counseling would lead patient to quit the habit. Further, $89.1 \%$ was in agreement that the tax on tobacco products need to be increased as a measure to reduce the SLT use.

Although the attitude change is a slow process, we have attempted to analyze the Likert scale attitudinal questions by converting to the dichotomized total $\mathrm{Z}$ scores. Total Z score were dichotomized: Less than zero as 'unsatisfactory attitudes' and above zero as 'satisfactory' attitude. However, pre assessment among the Nursing students was revealed that only 4 students were categorized as unsatisfactory attitudes and in the post assessment no one labeled as unsatisfactory attitudes (Table 2). Therefore, there is no significant difference between pre and post assessment.

\section{Discussion}

Tobacco cessation programmes are a "need of the hour" considering the widespread use and harmful effects of tobacco. However, most of the time emphasis is given for smoking cessation, reducing the significance of smokeless tobacco / areca nut (SLT/AN) use. Though it is accepted that both practices are harmful and thus the cessation programmes for both are equally important, novel strategies are required to overcome barriers and successfully implement such programmes. This is especially true for SLT/AN, as its use is traditionally ingrained in Sri Lankan and South Asian culture. Though, at a glance one would feel that time and energy spent on SLT/AN cessation programmes by healthcare professionals are not cost effective in terms of outcome, they do have a responsibility to find strategies to overcome the negativity. Thus the idea of conducting a series of workshops to enhance the knowledge of oral cancer and SLT/AN cessation practices among healthcare professionals was initiated.

The fact that nursing officers come in contact with a high numbers of patients make them ideal to deliver SLT/AN cessation counseling. However, it was found that tobacco cessation is not an integral component of their curricula. Hence it was essential to improve their knowledge and attitudes, related to SLT/AN cessation which was attempted with the present workshop. Results of the present study revealed a significant improvement in awareness of oral cancer as well as SLT/ AN cessation strategies of the participants (Figure 1 and 2) and thus workshops of this nature could be considered a cost effective method to improve awareness.

Nurses as part of healthcare professionals are in a position to educate patients regarding clinical presentations of oral cancer/OPMD, which may result in increased awareness among patients leading to early detection. The present study revealed that the nursing students were unaware of the main clinical presentations of oral cancer initially, with a significant improvement in post workshop knowledge. However, without creating an environment where this knowledge can be applied, the retention will be low as indicated by Wardh et al., (2008) [19]. In addition, though, the present study mainly focused on nursing students, it is possible to introduce such awareness programmes in the form of continuous professional development programmes to nursing officers currently working in hospitals, with the aim of recruiting them for the task of delivering tobacco cessation counseling.

Though, "manufacturing and selling of SLT products 
are banned in Sri Lanka”, only a few participants were aware of the fact. This reflects, that public health related messages of importance are not adequately transmitted to the population. Thus, it is mandatory to explore means to transmit relevant messages using social media or mobile apps, which may reach a higher proportion of younger generation, which comprised of majority of the current study population as well.

The knowledge related to tobacco cessation counseling was assessed by following statements. "Five " $R$ " concept can be used in counseling a client who is unwilling to quit SLT use" and "SLT users can be given existing drugs prescribed to combat cigarette craving". Both statements received low number of correct answers, prior to the workshop, which improved in the post workshop analysis. However, an improvement in knowledge does not directly translate into gaining of skills or confidence to practice tobacco cessation counseling. Thus further programmes are necessary with strategies such as role play to enhance tobacco cessation counseling skills, as indicated by the study population itself.

The present study did not analyze the tobacco usage practices of the study population. However, it can be concluded that tobacco usage was very low among female nursing students which comprised of majority of the study sample, due to cultural influences. Thus they are better suited to give SLT/AN cessation advice, because according to literature outcome is better when tobacco cessation advice is provided by non-tobacco users [20].

A Cochrane review by Rice et al., (2017) [21], showed moderate quality evidence to support that advice and support from nurses could increase people's success in quitting smoking, whether in hospitals or in community settings. Though, literature does not reveal any studies related to SLT/AN cessation programmes conducted by nurses, another Cochrane review by Ebbert et al., (2015) summarizes that both pharmacological and behavioral interventions may help SLT users to quit. In contrast, Nethan et al., (2018), in their systematic review indicates that behavioral interventions alone showed high efficacy in SLT cessation among adults with quit rate between 9 - 51.5 per cent, at six months. Thus, these evidences are sufficient to initiate SLT/AN related behavioral interventions by nurses as a first step [22-23].

The time available and focus on acute care in a hospital set up may act as a barrier for effective SLT/AN cessation counseling. However, studies have shown that effective tobacco cessation counseling could even be given at emergency care departments [24-25].

Majority of the nursing students who took part in the study believed that TCC should be a part of the regular treatment modalities and that proper counseling would lead patient to quit the habit. Thus it is timely to consider these sentiments of positivity and incorporate nursing fraternity to the SLT/AN cessation counseling task force, after formulating policy decisions.

The workshop was conducted using a series of lectures. Therefore, though there is an improvement in knowledge, participants may not have been able to gain skills and confidence required to deliver successful SLT/
AN counseling via the present workshop, which could be considered as a limitation. Thus continuous training programs aimed at acquiring above mentioned skills will be a future requirement.

In conclusion, workshops are a cost effective method to improve awareness related to SLT/AN cessation counseling. Nursing staff either at hospital or community setting would be an ideal choice to deliver such advice. Further, this model is very useful in the low economical settings.

\section{Acknowledgements}

Grant from the Presidential Task Force on Drug Prevention is highly appreciated.

\section{References}

1. Scientific Committee on Emerging and Newly Identified Health Risks SCENIHR. (2008). Health Effects of Smokeless Tobacco Products. Retrieved from https:// ec.europa.eu/health/ph_risk/committees/04_scenihr/docs/ scenihr_o_013.pdf..

2. Sinha D, Galapatti K, Rinchen S, Kahandaliyanage A, Mehta F, Jayasuriya-Dissanayake N, Somatunga L, Sumanasekera P. Smokeless tobacco use in Sri Lanka. Indian Journal of Cancer. 2012;49(4):357. https://doi.org/10.4103/0019509x.107729

3. Ministry of Healthcare and Nutrition Sri Lanka. (2009). Brief Profile on Tobacco Control in Sri Lanka. Retrieved from http://origin.searo.who.int/tobacco/documents/2009-pub3. pdf..

4. Cancer Registry National Cancer Control Programme, Colombo 05 Sri Lanka. (2009). Cancer Incidence Data: Sri Lanka Year 2001-2005, 7th Edition [Dataset]. Retrieved from https://www.nccp.health.gov.lk/pdf/publications/ cancer_incidece/Cancer_Incidence_Data_2005.pdf..

5. Amarasinghe HK, Usgodaarachchi US, Johnson NW, Lalloo R, Warnakulasuriya S. Betel-quid chewing with or without tobacco is a major risk factor for oral potentially malignant disorders in Sri Lanka: A case-control study. Oral Oncology. 2010 04;46(4):297-301. https://doi.org/10.1016/j. oraloncology.2010.01.017

6. Amarasinghe HK, Usgodaarachchi US, Johnson NW, Lalloo R, Warnakulasuriya S. Public awareness of oral cancer, of oral potentially malignant disorders and of their risk factors in some rural populations in Sri Lanka. Community Dentistry and Oral Epidemiology. 201008 23;38(6):540-548. https:// doi.org/10.1111/j.1600-0528.2010.00566.x

7. Ariyawardana A, Sitheeque MAM, Ranasinghe AW, Perera I, Tilakaratne WM, Amaratunga EAPD, Yang Y, Warnakulasuriya S. Prevalence of oral cancer and pre-cancer and associated risk factors among tea estate workers in the central Sri Lanka. Journal of Oral Pathology \& Medicine. 200708 01;36(10):581-587. https://doi.org/10.1111/j.16000714.2007.00583.x

8. Axell T, Holmstrup P, Kramer IRH, Pindborg JJ, Shear M. International seminar on oral leukoplakia and associated lesions related to tobacco habits. Community Dentistry and Oral Epidemiology. 1984 06;12(3):145-154. https://doi. org/10.1111/j.1600-0528.1984.tb01428.x

9. National cancer control programme, Sri Lanka. (2015). National Guideline for Management of Oral Potentially Malignant Disorders A Guide for Dental and Medical 
Practitioners (2nd ed.). Colombo, Sri Lanka: Department of Government Printing..

10. Saddichha S, Rekha D, Patil B, et al. Knowledge, attitude and practices of Indian dental surgeons towards tobacco control: Advances towards prevention. Asian Pac J Cancer Prev. 2010;11(4):939-42.

11. Albert D, Ward A, Ahluwalia K, Sadowsky D. Addressing Tobacco in Managed Care: A Survey of Dentists' Knowledge, Attitudes, and Behaviors. American Journal of Public Health. 2002 06;92(6):997-1001. https://doi.org/10.2105/ ajph.92.6.997

12. Mohanty VR, Rajesh GR, Aruna D. Role of Dental Institutions in Tobacco Cessation in India: Current Status and Future Prospects. Asian Pacific Journal of Cancer Prevention. 201304 30;14(4):2673-2680. https://doi. org/10.7314/apjcp.2013.14.4.2673

13. Chandrashekar J, Manjunath B, Unnikrishnan M. Addressing Tobacco Control in Dental Practice: A Survey of Dentists' Knowledge, Attitudes and Behaviors in India. Oral Health Prev Dent. 2011;9(3):243-9.

14. Overview | Smokeless tobacco: South Asian communities | Guidance | NICE. (2012, September 26). Retrieved from https://www.nice.org.uk/guidance/ph39..

15. Warnakulasuriya S. Effectiveness of tobacco counseling in the dental office. J Dent Educ. 2002;66(9):1079-87.

16. Carson KV, Verbiest ME, Crone MR, Brinn MP, Esterman AJ, Assendelft WJ, Smith BJ. Training health professionals in smoking cessation. . Cochrane Database of Systematic Reviews. 201205 16; https://doi.org/10.1002/14651858. cd000214.pub2

17. Benegal V, Rajkumar R, Muralidharan K. Does areca nut use lead to dependence? Drug and Alcohol Dependence. 200809 01;97(1-2):114-121. https://doi.org/10.1016/j. drugalcdep.2008.03.016

18. Mirza SS, Shafique K, Vart P, Arain MI. Areca nut chewing and dependency syndrome: Is the dependence comparable to smoking? a cross sectional study. Substance Abuse Treatment, Prevention, and Policy. 2011;6(1):23. https:// doi.org/10.1186/1747-597x-6-23

19. Wårdh I, Paulsson G, Fridlund B. Nursing staff's understanding of oral health care for patients with cancer diagnoses: an intervention study. Journal of Clinical Nursing. 200802 19;0(0):080219113329186-???. https:// doi.org/10.1111/j.1365-2702.2007.02051.x

20. Movsisyan NK, Varduhi P, Arusyak H, Diana P, Armen M, Frances SA. Smoking behavior, attitudes, and cessation counseling among healthcare professionals in Armenia. BMC Public Health. 2012 Nov 24;12(1). https://doi. org/10.1186/1471-2458-12-1028

21. Rice VH, Heath L, Livingstone-Banks J, Hartmann-Boyce J. Nursing interventions for smoking cessation. . Cochrane Database of Systematic Reviews. 2017 Dec 15;. https://doi. org/10.1002/14651858.cd001188.pub5

22. Ebbert JO, Elrashidi MY, Stead LF. Interventions for smokeless tobacco use cessation. Cochrane Database of Systematic Reviews. 2015 Oct 26; https://doi. org/10.1002/14651858.cd004306.pub5

23. Mehrotra R, Nethan S, Sinha D, Chandan K. Smokeless tobacco cessation interventions: A systematic review. Indian J Med Res. 2018;148(4):396.

24. Lowenstein SR, Koziol-McLain J, Thompson M, Bernstein E, Greenberg K, Gerson LW, Buczynsky P, Blanda M. Behavioral Risk Factors in Emergency Department Patients: A Multisite Survey. Academic Emergency Medicine. 1998 08;5(8):781-787. https://doi.org/10.1111/j.1553-2712.1998. tb02504.x
25. C. Bock, Bruce Becker, Raymond Niau B. Smoking among emergency chest pain patients: motivation to quit, risk perception and physician intervention. Nicotine \& Tobacco Research. 200002 01;2(1):93-96. https://doi. org/10.1080/14622200050011358

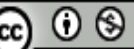

This work is licensed under a Creative Commons AttributionNon Commercial 4.0 International License. 